\title{
Entropy Generation Study of MHD Thermosolutal Convection in a Square Cavity for Different Prandtl Numbers
}

\author{
Mounir Bouabid*, Ammar Ben Brahim \\ Gabes University, Engineers National School of Gabes, Chemical and Process Engineering Department, Omar Ibn El Khattab Street, \\ 6029, Gabes, Tunisia
}

\begin{abstract}
Thermosolutal convection in a square cavity filled with Boussinesq fluid is numerically investigated. The cavity is heated along the active walls whereas the two other walls of the cavity are adiabatic and insulated. Entropy generation due to heat and mass transfer, fluid friction and magnetic effect has been determined in transient state for laminar flow by solving numerically the continuity, momentum energy and mass balance equations, using a Control Volume Finite-Element Method. The structure of the studied flows depends on six dimensionless parameters which are the Prandtl number, the thermal Grashof number, the buoyancy ratio, the Lewis number, the Hartman number and the inclination angle of the magnetic field. Results show that the magnetic field parameter has reducing the flow in the cavity and this lead to a decrease of entropy generation, Temperature decreases with increasing the value of the magnetic field parameter. The average Nusselt number increases with the Prandtl number and, in particular, its effect is more evident at high Hartmann numbers.
\end{abstract}

Keywords Thermosolutal Convection, Square Cavity, Entropy Generation; Magnetic Effect, Prandtl Number Effect

\section{Introduction}

Combined heat and mass transfers characteristics of thermosolutal convection with an external applied magnetic field to the cavity has received considerable attention lately. The externally imposed magnetic field is a widely used tool for control of melt flow in bulk crystal growth of semiconductors, application in engineering such as magnetic cooling, magnetic refrigerator, water treatment device, corrosion inhibition treatment, magneto hydrodynamics (MHD) power generation, plasma techniques and crystal growth. One of the main purposes of the electromagnetic control is stabilization of the flow and suppression of the oscillatory instabilities arising at certain values of the control parameters. The convection of electrically conducting fluids such as liquid metal in the presence of magnetic field has been one of the major interesting research subjects due to its direct application to various physical phenomena as well as to crystal growth processes. It is an established physical fact that the motion of an electrically conducting fluid is suppressed by the presence of a magnetic field. Also, some important crystal materials are good electrical conductors in

* Corresponding author:

bouabid.mpcshun@yahoo.fr (Mounir Bouabid)

Published online at http://journal.sapub.org/mechanics

Copyright (C) 2011 Scientific \& Academic Publishing. All Rights Reserved their liquid state.

The numerical studies of heat and mass transfers with magnetic effects reported in the literature are relatively scarce and invariably use as governing equations the Navier-Stokes and energy and mass equations. Entropy generation in thermosolutal convection through a square cavity is numerically calculated using the Control Volume Finite Element Method (CVFEM) which taken into consideration the effect of a Prandtl number variation in the range of $0.025 \leq \operatorname{Pr} \leq 7.1$, the application of the magnetic field reduces the convective heat transfer rate in the cavity for any inclined angle. It was established that the magnetic field suppresses the convection and forces it to move to the free surface. Magnetic field with the parallel to the free surface intensity vector splits the convection into the multi-layered structure.

Howard et al.[1] investigated a two-phase non-boiling slug flow regime for the purposes of enhancing heat transfer in micro channel heat sinks or compact heat exchangers. Varying Prandtl and Capillary numbers caused notable effects in the transition region between entrance and fully developed flows. Significant Nu oscillations were observed for low Pr fluids due to internal circulation within the slug. However, these oscillations are observed to be damped out when higher Prandtl number fluids are employed. Combined effect of Prandtl number and Richardson number on the wake dynamics and heat transfer is studied by Sarkar et 
al.[2]. In the case of forced convection, crowding of temperature contours with reduced spatial spread is observed for increasing Prandtl numbers. The local and average Nusselt numbers are found to increase with increasing Reynolds number and Prandtl number. Effect of Prandtl number shows various interesting phenomena for the mixed convective flows. Increasing the Prandtl numbers resulted in decreasing deflection and strength in the wake structures. The effect of increasing Prandtl number is manifested as the stabilizing effect in the flow. Dritselis and Vlachos.[3] are numerically studied the magnetic field effects on the coherent structures and the associated heat transfer in a turbulent channel flow with constant temperature at the bottom (cold) and top (hot) walls. Two fluids with Prandtl numbers of 0.01 and 0.71 are studied. It is shown that the conditionally averaged quasi-stream wise vortices are modified by the magnetic field with their size being increased and their strength decreased. The fluid motions are damped by the Lorentz force. For the higher Prandtl number fluid, a similarity between the coherent temperature and the coherent stream wise velocity fluctuations is observed for both types of flow. This is diminished for the lower Prandtl number fluid, especially in the magneto hydrodynamic flow, inhibiting the intrusion of cold (hot) fluid from the cold (hot) wall towards the central region. Yu et al are firstly.[4 investigated the Prandtl number effect on flow and heat transfer characteristics. It is found that the flow and heat transfer characteristics for a low Prandtl number fluid $(\mathrm{Pr}=0.03)$ are unique and they are almost independent of Prandtl number when $\operatorname{Pr} \geq 0.7$. The entire spectrum of Prandtl number investigated can be divided into three sections based on the variations of average heat transfer coefficients. In each section, correlating equations of the average Nusselt number to the Rayleigh number are proposed with the maximum deviation less than 3\%. Secondly.[5], they are interested to a liquid gallium $(\operatorname{Pr}=0.023)$ in their investigation of transient natural convective heat transfer of a low-Prandtl number fluid inside a horizontal circular cylinder with an inner coaxial triangular cylinder. The development of the convective flow and heat transfer is shown via the time histories of the average Nusselt number. It is found that the time-averaged Nusselt number is apparently increased by horizontally placing the top side of the inner triangular cylinder for $\mathrm{Gr} \geq 10^{5}$. Ould-Rouiss et al.[6] are investigated the effect of Prandtl number on the turbulent thermal statistics in fully developed annular pipe flow, with isoflux boundary conditions. The Prandtl number has marked influence on the thermal field. With decreasing Pr, the conductive sublayer at both walls spreads from the walls to the core region, while the root mean square of temperature fluctuations and the turbulent heat fluxes are reduced near both walls. Asymptotic behaviors of these quantities are analyzed.

Entropy generation in natural convection through an inclined rectangular cavity is numerically calculated using the Control Volume Finite Element Method (CVFEM) by Bouabid et al.[7]. Results show that total entropy generation increases with the aspect ratio of the cavity for high thermal Grashof number, for any fixed irreversibility distribution ratio and with the last parameter at constant Grashof number. Bouabid et al.[8] are then studied the contributions of thermal, diffusive, friction and magnetic terms on entropy generation are investigated. The more effect was due to heat transfer and then to mass transfer. They obtained that the magnetic effect is more pronounced than friction one. The magnetic field parameter suppresses the flow in the cavity and this lead to a decrease of entropy generation. At local level, results show that entropy generation lines are localized on lower heated and upper cooled regions of the active walls. Lage and Bejan.[9] are studied laminar natural convection in a square enclosure heated through the side walls for $0.01 \leq \operatorname{Pr} \leq 10$ and $10^{2} \leq \mathrm{Ra} \leq 10^{11}$ and addressed the influence of the Prandtl number on the heat transfer. Jalil and Al-Tae'y.[10] are numerically studied the effect of the direction of external magnetic field applied on liquid metal (molten sodium) fills a square enclosure. They showed that a magnetic field in $\mathrm{x}$-direction is more effective on flow pattern and temperature distribution than from y-direction and the orientation effect of magnetic field depends on which magnetic field component $\mathrm{B}_{\mathrm{x}}$ or $\mathrm{B}_{\mathrm{y}}$ is in demand. The value of Nusselt number decreases with increase in Hartmann number value at same orientation of magnetic field, and the change in Nusselt number value with magnetic field orientation dependent on which value of electromagnetic force is in demand in $\mathrm{x}$-direction or $\mathrm{y}$-direction. The Control of the instability by a magnetic field was done by Davoust et al.[11] in order to reach the following goals: to promote stabilization or destabilization of a thermo gravitational Hadley recirculating flow, free of any Marangoni effects, in a cylinder filled with mercury and submitted to a uniform vertical magnetic field $\mathrm{B}_{0}$. They obtained that temperature oscillations are found to occur with reproducibility when $\mathrm{B}_{0}$ is smoothly decreased. At a moderate scale of the Grashof number (i.e. $\mathrm{Gr}_{\mathrm{T}}=10^{5}$ ), oscillatory instabilities occur by way of a subcritical bifurcation which yields two waves. Al-Najem et al.[12] used the power control volume approach to determine the flow and temperature fields under a transverse magnetic field in a titled square enclosure with isothermal vertical walls and adiabatic horizontal walls at Prandtl number of 0.71 . They showed that the suppression effect of the magnetic field on convection currents and heat transfer is more significant for low inclination angles and high Grashof numbers. Effect of directions of magnetic field (i.e. radial or axial) on the buoyancy-driven convection in a vertical cylindrical annulus filled with a low Prandtl number electrically conducting fluid $(\mathrm{Pr}=0.054)$ was examined by Sankar et al.[13], they found that the external magnetic field in the vertical direction was found to be effective than the magnetic field applied parallel to the heated vertical wall, the magnetic field suppresses the convective flow and eliminates the flow oscillations. The direction of magnetic field plays an important role in suppressing the convective flows. The magnetic field is more effective when it is perpendicular to the direction of the primary flow. 
This phenomenon has a serious implication on the design of magnetic systems for stabilizing or weakening the convective effects. Laminar, two-dimensional MHD natural convection within a liquid gallium filled square enclosure in the presence of inclined magnetic field was investigated by Sathiyamoorthy and Chamkha.[14], the application of the magnetic field reduces the convective heat transfer rate in the cavity for any inclined angle. In addition, the local Nusselt number at the bottom wall of the cavity exhibited oscillatory behavior along the horizontal distance for the case of linearly heated side walls whereas it increased continuously for the case of linearly heated left wall and cooled right wall with the exception of large Hartmann numbers for a vertically-applied magnetic field. The average Nusselt numbers for the bottom and side walls for the case of linearly heated side walls showed an oscillatory behavior with increasing values of the Hartmann numbers especially for a vertically-applied magnetic field whereas the average Nusselt numbers for the bottom, left and right walls for the case of linearly heated left wall and cooled right wall decreased as the Hartmann number was increased. Pesso and Piva.[15] are numerically investigated the laminar natural convection in a square cavity heated through the side walls at low Prandtl numbers with large density differences. They concluded that the mean Nusselt number increases with the Prandtl number and, in particular, its effect is more evident at high Rayleigh numbers. Entropy generation in convective heat and mass transfer through an inclined enclosure is numerically investigated by Magherbi et al.[16]. They showed that entropy generation increases with the thermal Grashof number and the buoyancy ratio for moderate Lewis number. At local level, irreversibility due to heat and mass transfer are nearly identical and are localized in the bottom heated and the top cooled walls of the enclosure. The inclination angle of the cavity has more effect on entropy generation for thermal Grashof number $\mathrm{Gr}_{\mathrm{T}} \geq 10^{4}$. In this case, irreversibility increases towards a maximum value obtained for an angle $\sim 45^{\circ}$, then decreases and tends towards unity value for inclination angle $\sim 180$.

The aim of this paper is to examine the effect of a Prandtl number variation on entropy generation in combined convective heat and mass transfers in the studied cavity under an applied externally magnetic field.

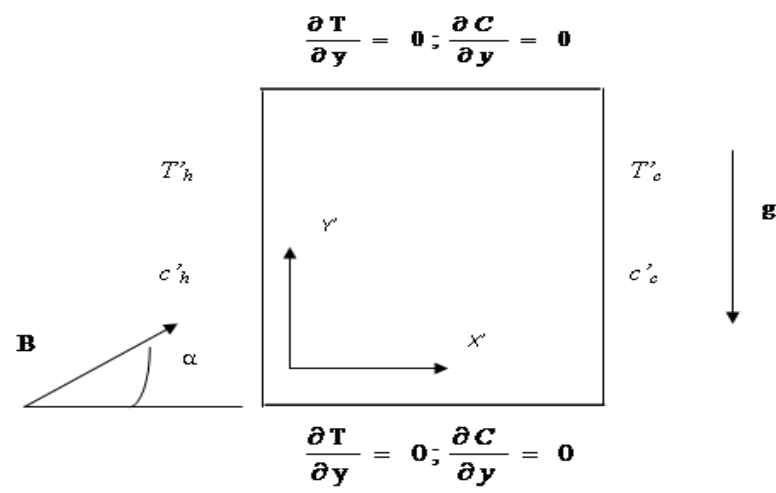

Figure 1. Schematic view of the physical model.

\section{Governing Equations}

We consider a 2D square cavity submitted to an external oriented magnetic field, $\mathrm{B}$ with an inclination angle, $\alpha$ as shown in Figure 1.

The two active left and right walls are at different but uniform temperatures and concentrations $\left(\mathrm{T}_{\mathrm{h}}, \mathrm{C}_{\mathrm{h}}{ }_{\mathrm{h}}\right)$ while the two other walls are insulated and adiabatic. The fluid is considered as a Newtonian, Boussinesq incompressible fluid, their properties are described by its kinematic viscosity $v$, its thermal diffusivity and its thermal and solutal volumetric expansion coefficients $\beta_{\mathrm{T}}$ and $\beta_{\mathrm{C}}$. The mass density of the fluid is considered to vary linearly with temperature and concentration such as:

where:

$$
\rho=\rho o\left[1-\beta_{\mathrm{T}}(\mathrm{T}-\mathrm{To})-\beta_{\mathrm{C}}(\mathrm{C}-\mathrm{C} \text { o })\right]
$$

$$
\begin{aligned}
& \beta_{T}=-\frac{1}{\rho_{o}}\left(\frac{\partial \rho}{\partial T}\right)_{P} \\
& \beta_{C}=-\frac{1}{\rho_{o}}\left(\frac{\partial \rho}{\partial C}\right)_{P}
\end{aligned}
$$

The set of the dimensionless governing equations is, given by:

$$
\begin{gathered}
\frac{\partial u}{\partial x}+\frac{\partial v}{\partial y}=0 \\
\frac{\partial u}{\partial t}+\operatorname{div} J_{u}=-\frac{\partial P}{\partial x}+\operatorname{Pr} \cdot H a^{2}(v \cos \alpha-u \sin \alpha) \sin \alpha \\
\frac{\partial v}{\partial t}+\operatorname{div} J_{v}=-\frac{\partial P}{\partial y}+G r \cdot(T+N \cdot C)+\operatorname{Pr} \cdot H a^{2}(u \sin \alpha-v \cos \alpha) \cos \alpha \\
\frac{\partial T}{\partial t}+\operatorname{div} J_{T}=0 \\
\frac{\partial C}{\partial t}+\operatorname{div}_{C}=0
\end{gathered}
$$

with:

$$
\begin{gathered}
J_{u}=u \cdot V-\operatorname{Pr} \operatorname{grad} u ; J_{v}=v \cdot V-\operatorname{Pr} \operatorname{grad} v \\
J_{T}=T . V-\operatorname{grad} T ; J_{C}=C \cdot V-\frac{1}{L e} \operatorname{grad} C
\end{gathered}
$$

where the dimensionless variables are defined by:

$$
\begin{gathered}
x=\frac{x^{\prime}}{L} ; \quad y=\frac{y^{\prime}}{L} ; \quad u=\frac{u^{\prime}}{U^{*}} ; \quad v=\frac{v^{\prime}}{U^{*}} ; \quad t=\frac{t^{\prime} U^{*}}{L} \\
p=\frac{p^{\prime}}{\rho_{0} U^{* 2}} ; \quad T=\frac{T^{\prime}-T^{\prime} 0}{\Delta T} ; \quad C=\frac{C^{\prime}-C^{\prime} 0}{\Delta C} \\
U^{*}=\frac{\alpha_{T}}{L} ; \quad \lambda=\rho \alpha_{T} C_{p} \\
\operatorname{Pr}=\frac{v}{\alpha_{T}} ; G r_{T}=\frac{g \beta_{T} \cdot \Delta T \cdot L^{3}}{v^{2}} ; G r_{C}=\frac{g \beta_{C} \cdot \Delta C \cdot L^{3}}{v^{2}} \\
N=\frac{\beta_{C} \cdot G r_{C}}{\beta_{T} \cdot G_{T}} ; H a \sqsubset \frac{\mathrm{T}_{e} B L}{\mu} L e=\frac{\alpha_{T}}{D}
\end{gathered}
$$

The appropriate initial and boundary conditions to the 
laminar flow within the cavity are:

at, $\mathrm{t}=0$ for whole space:

$\mathrm{u}=\mathrm{v}=0 ; \mathrm{P}=0 ; \mathrm{T}=0.5-\mathrm{x}$ and $\mathrm{C}=0.5-\mathrm{x}(17)$

Adiabatic walls:

$$
\frac{\partial \phi}{\partial y}=0 \text { On plane } \mathrm{y}=0 \text { and } \mathrm{y}=1
$$

Active walls:

$$
\begin{array}{cc}
\phi=0.5 & \text { On plane } \mathrm{x}=0 \\
\varphi=-0.5 & \text { On plane } \mathrm{x}=1
\end{array}
$$

$\varphi$ : Physical parameter representing temperature or concentration.

\section{Formulation}

Governing Equations (4)-(8) could be solved for the determination of the temperature, the concentration and the velocity scalar fields which depend on the choice of the numerical support of resolution. In this study, a Control Volume Finite Element Method (CVFEM) of Saabas and Baliga.[17] is used. A standard grid at which diagonals are added to form triangular elements around each node where velocity components are calculated is considered. For pressure, temperature and concentration scalar field a staggered grid is used. Pressure and the other scalar field are calculated at different points to avoid the problem of oscillations.

The system of the governing equations is resolved by applying the SIMPLE algorithm of Patankar.[18]. The SIMPLER algorithm and the SIMPLEC approximation of Van Doormal and Raithby are used in conjunction with an Alternating Direction Implicit (ADI) Scheme for performing the time evolution. The used numerical Code written in FORTRAN language was described and validated in details in Abbassi et al.[19, 20].

The existence of thermal and diffusive gradients between the active walls of the cavity, in addition to magnetic field effects, set the fluid in a non-equilibrium state which causes entropy generation in the system. According to local thermodynamic equilibrium with linear transport theory, the local entropy generation is given by.[21]:

$$
\begin{aligned}
& \dot{S}_{\text {gen }}=\frac{\lambda\left(\operatorname{grad} T^{\prime}\right)^{2}}{T^{\prime 2}}-\frac{1}{T^{\prime}} \sum_{i} J_{\alpha^{\prime} i} \operatorname{grad} \mu_{i} \\
& =\overline{\bar{\tau}: \overline{\overline{\operatorname{grad}}} V} \frac{\sigma|V \times B|^{2}}{T^{\prime}}+\frac{\sigma \mid}{T^{\prime}}
\end{aligned}
$$

$J_{\alpha^{\prime} i}$ and $\mu_{i}$ are mass diffusion flux of species $i$ in phase $\alpha$ ' and its chemical potential, respectively. As it can be seen from Equation (14), the right hand side this equation represents four terms which are: Irreversibility due to heat transfer, the second is due to mass transfer, the third is due to fluid friction and the fourth is due to magnetic force.

For a two dimensional flow and by assuming bulk concentration $\left(\mathrm{C}^{\prime}{ }_{0}\right)$ and temperature $\left(\mathrm{T}^{\prime}{ }_{0}\right)$ in the denominator of Equation (21) with a single diffusing species, Equation (21) can therefore be written as follows:

$$
\begin{aligned}
& \dot{S}_{g e n}=\frac{\lambda}{T_{0}{ }^{2}}\left(\operatorname{grad} T^{\prime}\right)^{2}+\frac{R D}{C_{0}^{\prime}}\left(\operatorname{grad} C^{\prime}\right)^{2} \\
& +\frac{R D}{\mathrm{~T}_{o}^{\prime}}\left[\left(\operatorname{grad} C^{\prime}\right) \cdot\left(\operatorname{grad} T^{\prime}\right)\right] \\
& +\frac{\mu}{T_{0}{ }^{\prime}}\left[2\left(\frac{\partial u^{\prime}}{\partial x^{\prime}}\right)^{2}+2\left(\frac{\partial v^{\prime}}{\partial y^{\prime}}\right)^{2}+\left(\frac{\partial u^{\prime}}{\partial y^{\prime}}+\frac{\partial v^{\prime}}{\partial x^{\prime}}\right)^{2}\right] \\
& +\frac{\sigma B^{2}}{T_{0}{ }^{\prime}}(u \sin \alpha-v \cos \alpha)^{2}
\end{aligned}
$$

The local entropy generation can be putted in a dimensionless form by using the dimensionless variables listed in Equations (11)-(16) in the following way:

$$
\mathrm{N}_{s, \ell}=\mathrm{N}_{h}+\mathrm{N}_{f}+\mathrm{N}_{d}^{C . C}+\mathrm{N}_{d}^{T . C}+\mathrm{N}_{B}
$$

where:

$$
\begin{gathered}
\mathrm{N}_{h}=\left[\left(\frac{\partial \mathrm{T}}{\partial x}\right)^{2}+\left(\frac{\partial \mathrm{T}}{\partial y}\right)^{2}\right] \\
\mathrm{N}_{f}=\lambda_{1}\left[2\left(\frac{\partial u}{\partial x}\right)^{2}+2\left(\frac{\partial v}{\partial y}\right)^{2}+\left(\frac{\partial v}{\partial x}+\frac{\partial u}{\partial y}\right)^{2}\right] \\
\mathrm{N}_{d}^{C . C}=\lambda_{2}\left[\left(\frac{\partial C}{\partial x}\right)^{2}+\left(\frac{\partial C}{\partial y}\right)^{2}\right] \\
\mathrm{N}_{d}^{T . C}=\lambda_{3}\left[\left(\frac{\partial \mathrm{T}}{\partial x}\right)\left(\frac{\partial C}{\partial x}\right)+\left(\frac{\partial \mathrm{T}}{\partial y}\right)\left(\frac{\partial C}{\partial y}\right)\right] \\
\mathrm{N}_{\mathrm{B}}=\lambda_{4} \cdot(u \sin \alpha-v \cos \alpha)^{2}
\end{gathered}
$$

with:

$$
\begin{aligned}
& \lambda_{1}=\frac{\mu}{\lambda} T_{0}^{\prime}\left(\frac{\alpha_{T}}{L \Delta T}\right)^{2} ; \lambda_{2}=\frac{R D T_{0}^{\prime}}{\lambda \cdot C_{0}^{\prime}}\left(\frac{\Delta C}{\Delta T}\right)^{2} \\
& \lambda_{2}=\frac{R D T_{0}^{\prime}}{\lambda}\left(\frac{\Delta C}{\Delta T}\right) \quad \lambda_{B}=\frac{\sigma T_{0}^{\prime}}{\lambda}\left(\frac{B \alpha_{T}}{\Delta T}\right)^{2}
\end{aligned}
$$

where $\mathrm{N}_{h}, \mathrm{~N}_{f}, \mathrm{~N}_{d}^{C . C}, \mathrm{~N}_{d}^{T . C}$ and $\mathrm{N}_{B}$ are defined as local dimensionless entropy generation due to heat transfer $\left(\mathrm{S}_{\mathrm{th}}\right)$, fluid friction $\left(\mathrm{S}_{\mathrm{vis}}\right)$, mass transfer $\left(\mathrm{S}_{\mathrm{diff}}\right)$ by pure concentrations gradients, mass transfer by mixed product of concentration and thermal gradients and magnetic field $\left(\mathrm{S}_{\mathrm{mag}}\right)$, respectively.

$\lambda_{1}, \lambda_{2}, \lambda_{3}$ and $\lambda_{4}$ are irreversibility distribution ratios related to velocity gradients, concentrations gradients, mixed product of concentration and thermal gradients and magnetic field, respectively.

Total dimensionless entropy generation is obtained by a numerical integration of dimensionless local entropy generation through the entire volume of the cavity, $\Omega$. That is:

$$
S=\int_{\Omega} \cdot \mathrm{N}_{S . l} \cdot d \Omega
$$

The temperature and concentration gradients are computed through the heat wall of the cavity, and then used to calculate the average Nusselt and Sherwood numbers, they are given respectively by:

$$
\overline{\mathrm{N} u}=\int_{0}^{1}\left(-\frac{\partial T}{\partial y}\right) \cdot d x
$$


and:

$$
\overline{S h}=\int_{0}^{1}\left(-\frac{\partial C}{\partial y}\right) \cdot d x
$$

\section{Results and Discussions}

The Prandtl number, the thermal Grashof number, the buoyancy ratio, the irreversibility distribution ratios and the inclination angle of the magnetic field are in the following ranges: $0.025 \leq \operatorname{Pr} \leq 7.1 ; 10^{3} \leq \mathrm{Gr}_{\mathrm{T}} \leq 10^{5},-6 \leq \mathrm{N} \leq 6,10^{-7} \leq$ $\lambda_{f} \leq 10^{-4}$ and $0^{\circ} \leq \alpha \leq 180^{\circ}$. Grid of sizes of $51 \times 51$ nodal points and a step time, $\Delta \mathrm{t}=10^{-4}$ for all the studied thermal Grashof numbers are used.

Figure 2 shows the influence of the Prandtl number on the transient entropy generation for the case of natural convection (i.e., $\mathrm{N}=0$ ) and for relatively the absence of a magnetic effect $(\mathrm{Ha}=0)$. In this case, entropy generation quickly passes from a minimum value at the very beginning of the transient state towards a maximum value, and then exhibits an oscillatory behavior before reaching a constant value in steady state. Entropy generation increases with the increasing of the Prandtl number.

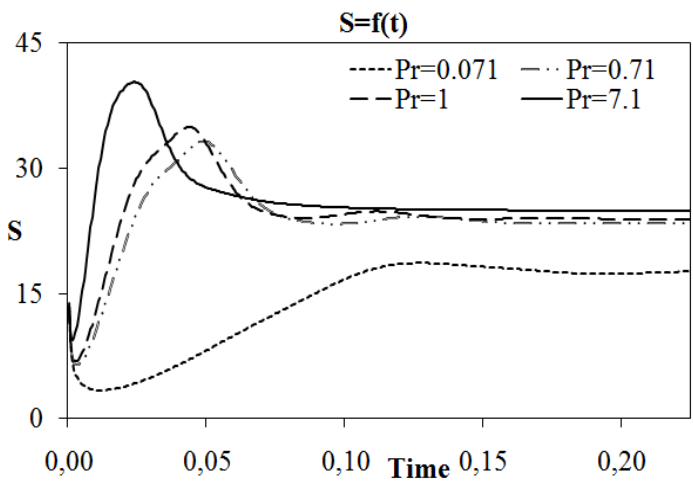

Figure 2. Dimensionless total entropy generation versus time for different Prandtl numbers at $\mathrm{Ha}=0 ; \mathrm{Gr}_{\mathrm{T}}=10^{5}$ and $\alpha=0^{\circ}$.

The variation of Nusselt number, $\mathrm{Nu}$ with Prandtl number, Pr for Newtonian fluids at two Hartmann number values ( $\mathrm{Ha}=0$ and $\mathrm{Ha}=25$ ) is illustrated in Figure 3(a), which shows that $\mathrm{Nu}$ decreases while increasing the magnetic effect and it increases with increasing Pr until reaching 0.71 value and then its variation has no great significance. It is clear that Pr has an important influence on $\mathrm{Nu}$ for small values (i.e., $\mathrm{Pr}<0.71$ ). However, $\mathrm{Nu}$ is relative insensitive to $\operatorname{Pr}$ for moderate Prandtl number values (i.e., $0.71<\operatorname{Pr}<$ 1). For high values of Prandtl number (i.e., $\operatorname{Pr}>1$ ), Prandtl number influence on Nusselt number is more pronounced than the previous case. In the present configuration, the relative strengths of inertial, viscous, magnetic and thermal forces determine the flow behavior. For small values of $\mathrm{Pr}$ the thermal boundary layer thickness remains much greater than the hydrodynamic boundary layer thickness. As a result of this difference, the transport behavior in the majority of the domain is governed by the inertial and thermal forces. In contrast, for large values of Pr, the hydrodynamic boun- dary layer thickness remains much greater than the thermal boundary later thickness thus the transport characteristics are primarily driven by thermal and viscous forces. For $\operatorname{Pr}>$ 1 , an increase in Pr decreases the thermal boundary layer thickness in comparison to the hydrodynamic boundary layer thickness. This change essentially acts to increase the heat flux which is reflected in the increasing of Nusselt number. In the case of $\operatorname{Pr}>0.71$, a change in Prandtl number principally modifies the relative balance between viscous and thermal forces so the heat transport in the thermal boundary layer gets only marginally affected. This modification is reflected in the weak Prandtl number dependence of $\mathrm{Nu}$ for moderate and large values of Pr. Flow characteristics domain can be then divided into three regions: Region. I (i.e. $\operatorname{Pr}<0.71$ ), region. II (i.e. $0.71<\operatorname{Pr}<1$ ) and region. III (i.e. $\operatorname{Pr}>1)$.

Figure 3(b) exhibits the profile of total entropy generation with the buoyancy ratio for different Prandtl numbers which depicted a minimum value at $\mathrm{N}=-1$. It decreases as well as the Prandlt number increases. As $\mathrm{N}$ increases $(\mathrm{N}>-1)$ on the right hand side of this minimum, the total entropy generation increases. The opposite trend is observed at the left hand side of the minimum $(\mathrm{N}<-1)$.

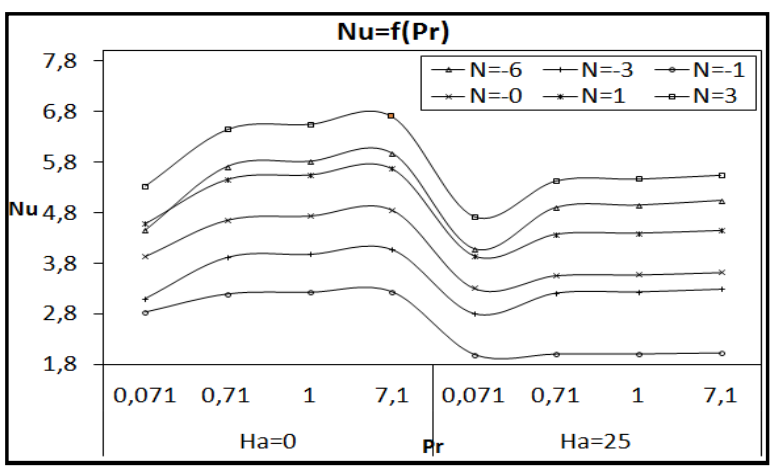

(a)

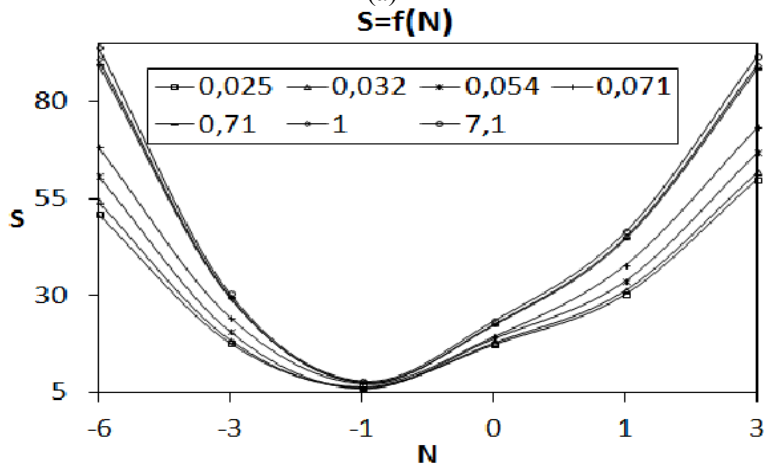

(b)

Figure 3. (a) Average Nusselt number versus Prandtl number for different buoyancy ratios at $\mathrm{Ha}=0$ and $\mathrm{Ha}=25$ (b) Dimensionless total entropy generation versus buoyancy ratio for different Prandtl numbers: $\mathrm{Gr}_{\mathrm{T}}=10^{5}$; $\mathrm{Ha}=25$ and $\alpha=0^{\circ}$.

Let us focus our interest to the minimum value of entropy generation on analyzing the variations of either Nusselt or Sherwood numbers with Prandtl and Hartmann numbers. Figure 4 (a) shows that the more significant variation of Nusselt number was found between 0.071 and 0.71.The 
same behavior was shown in Figure 4 (b) according to Sherwood profile. As Hartmann number increases these effects are less and less pronounced. Magnetic effect decreases entropy generation in the studied system by reducing the flow according to the resistant effect to the flow offered by Lorentz force. Concerning the entropy generation variation, figure 4 (c) exhibits an elongation of the Prandtl number variation range which has a sensitive effect to $0.054 \leq \operatorname{Pr} \leq 7.1$.Thus both low Prandtl number and high once must be taken into consideration in studying such convection problems.

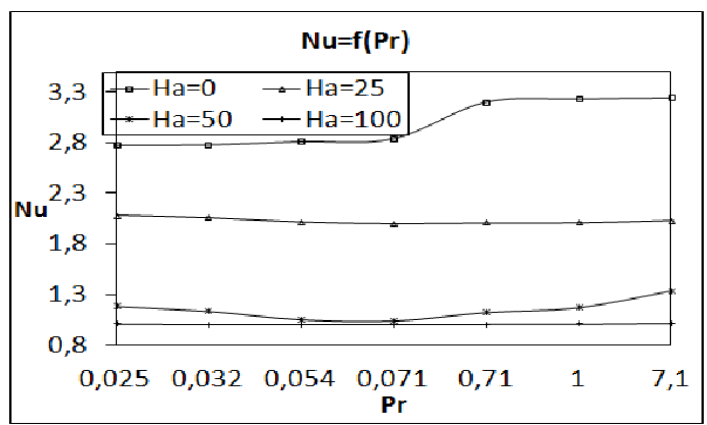

(a)

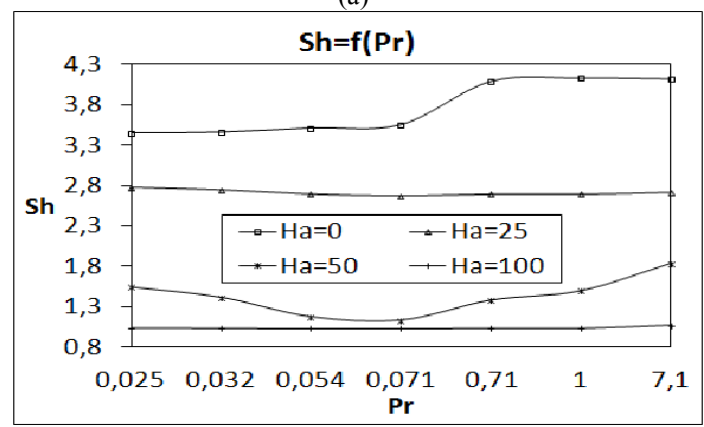

(b)

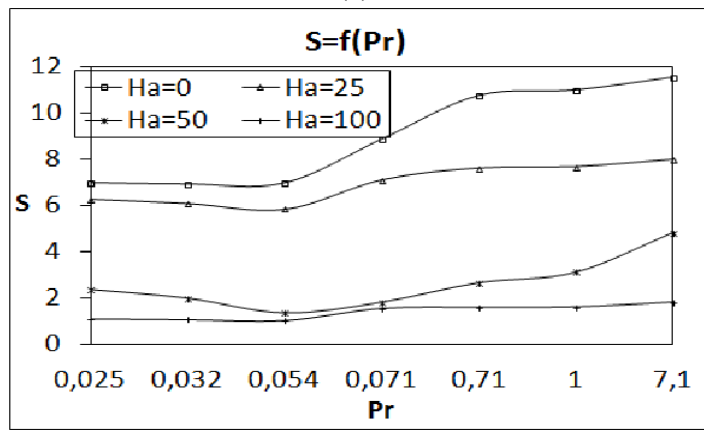

(c)

Figure 4. (a) Average Nusselt number (b) Average Sherwood number (c) Dimensionless total entropy generation versus Prandtl number for different Hartmann numbers: $\mathrm{Gr}_{\mathrm{T}}=10^{5} ; \mathrm{N}=-1$ and $\alpha=0^{\circ}$.

Figure 5 illustrates the behaviors of Maximum velocity components, $\mathrm{u}_{\max }$ and $\mathrm{v}_{\max }$. These profiles show a dependence on Prandtl number and buoyancy ratio variations. It can be noticed from figure 5(a) that the more effect of Prandtl number is obtained in the first region where $\mathrm{u}_{\max }$ velocity component tends to decrease as the strength of the Prandtl number increases for low magnetic effect $\mathrm{Ha}=25$. As depicted by figure $5(\mathrm{~b}), \mathrm{v}_{\max }$ velocity component increases for Prandtl number increasing. Both $u_{\max }$ and $v_{\max }$ profiles exhibit less significant effect for high Prandtl number values. The range of significant effect of Prandtl number was obtained as: $0.054 \leq \operatorname{Pr} \leq 0.71$.

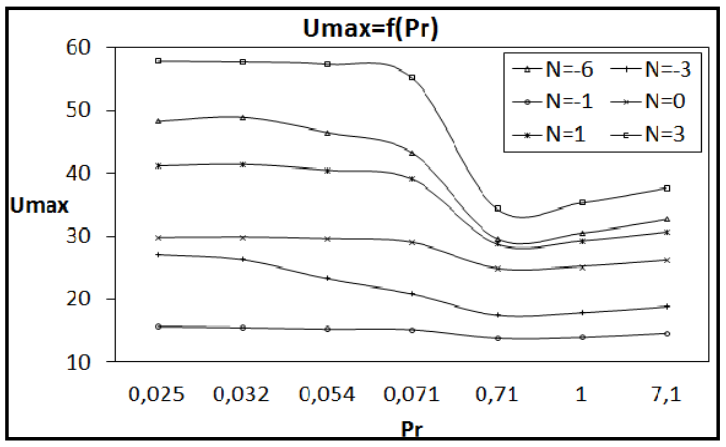

(a)

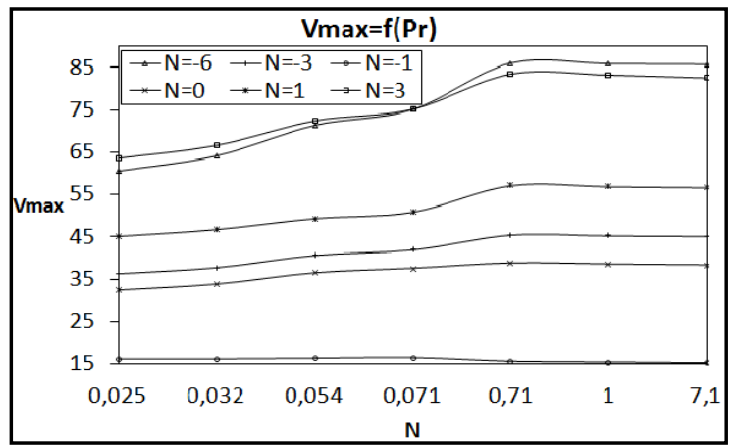

(b)

Figure 5. (a) Maximum x-component velocity (b) Maximum y-component velocity versus Prandtl number for different buoyancy ratios: $\mathrm{Gr}_{\mathrm{T}}=10^{5} ; \mathrm{Ha}=25$ and $\alpha=0^{\circ}$.

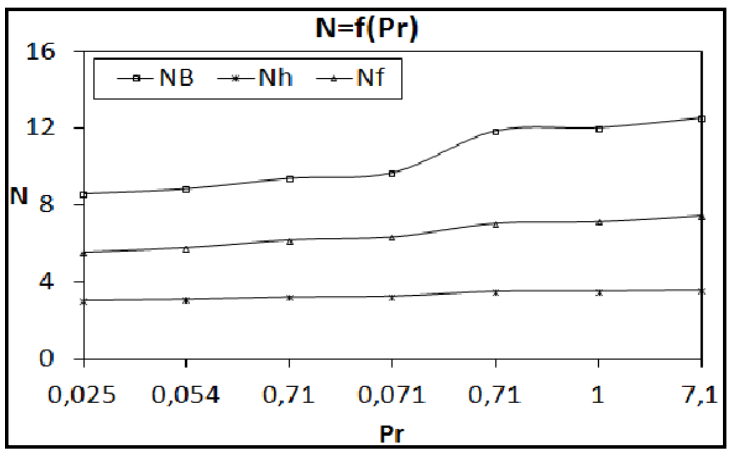

(a)

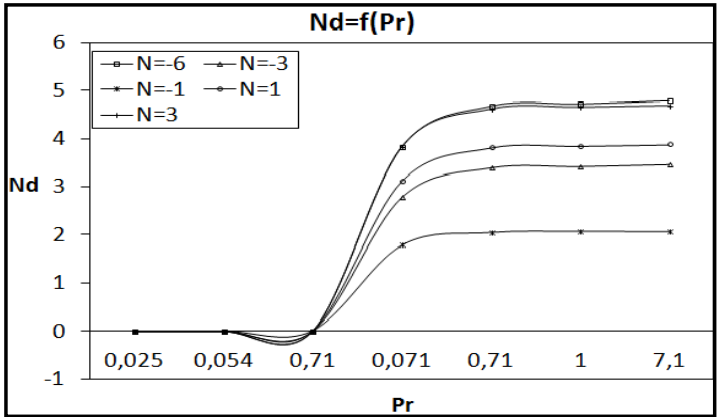

(b)

Figure 6. Thermal, friction and magnetic entropy generation variation for $\mathrm{N}=0$ and $\mathrm{Ha}=25$ (b) diffusive entropy generation variation versus Prandtl number for different buoyancy ratio at $\mathrm{Ha}=0: \mathrm{Gr}_{\mathrm{T}}=10^{5}$ and $\alpha=0^{\circ}$.

Different origins of entropy generation are illustrated in 
figure 6(a): thermal, friction and magnetic contribution at natural convection and the diffusive entropy generation term in figure $6(\mathrm{~b})$ in the absence of the magnetic effect, respectively. It can be noticed that thermal and friction irreversibilities are smaller than magnetic one. Their effects are more pronounced at high values of Prandtl number and essentially between 0.071 and 0.71 . For diffusive entropy generation, it is clear from figure 6(b) that for low Prandtl numbers, it has no effect but its influence start from 0.71 value of Prandtl number which depends on the buoyancy value.

\section{Conclusions}

Entropy generation due to thermosolutal convection in a square cavity is numerically calculated using the Control Volume Finite-Element Method (CVFEM). Results show that the total entropy generation, for natural convection, entropy generation quickly passes from a minimum value at the very beginning of the transient state towards a maximum value, and then exhibits an oscillatory behavior before reaching a constant value in steady state. Entropy generation increases with the increasing of the Prandtl number, Nusselt number behavior depends on the Prandtl number region; it is more sensitive to low values of Prandtl number. The Prandtl number will increase the Nusselt number, but it will decrease the wall friction coefficient. The presence of a magnetic field tends to reduce entropy generation where the system passes from an oscillatory behavior describing non linear branch of irreversible process towards an asymptotic behavior showing the linear branch of thermodynamics for irreversible processes. The profile of total entropy generation with the buoyancy ratio for different Prandtl numbers which depicted a minimum value at $\mathrm{N}=-1$. Behaviors of Maximum velocity components, $\mathrm{u}_{\max }$ and $\mathrm{v}_{\max }$ show a dependence on Prandtl number and buoyancy ratio variations.

\author{
$\mathrm{Nu}$ Nusselt number \\ $\mathrm{N}_{s, \ell}$ dimensionless local entropy generation \\ $S$ dimensionless total entropy generation \\ $\mathrm{P} \quad$ pressure $\left(\mathrm{kg} \cdot \mathrm{m}^{-1} \cdot \mathrm{s}^{-2}\right)$ \\ Pr Prandtl number \\ $\mathrm{Ra}_{\mathrm{T}}$ Rayleigh number \\ Sc Schmidt number \\ Sh Sherwood number \\ $S_{g e n}$ local volumetric entropy generation $\left(\mathrm{J} \cdot \mathrm{m}^{-3} \cdot \mathrm{s}^{-1} \cdot \mathrm{K}^{-1}\right)$ \\ $\mathrm{T}$ dimensionless temperature \\ $\mathrm{T}$ ' temperature $(\mathrm{K})$ \\ t' time (s) \\ $\mathrm{t}$ dimensionless Time \\ $\mathrm{T}_{\mathrm{h}}$ ' hot side temperature $(\mathrm{K})$ \\ $\mathrm{T}_{\mathrm{c}}$ c cold side temperature $(\mathrm{K})$ \\ $\mathrm{T}^{\prime}$ o bulk temperature $(\mathrm{K})$ \\ $\mathrm{u}, \mathrm{v}$ dimensionless velocity components \\ $\mathrm{U}^{*} \quad$ characteristic Velocity $\left(\mathrm{m} \cdot \mathrm{s}^{-1}\right)$ \\ $\mathrm{V}$ velocity vector $(\mathrm{m} \cdot \mathrm{s}-1)$ \\ u', v' velocity components along $x^{\prime}, y^{\prime}$ respectively $\left(\mathrm{m} \cdot \mathrm{s}^{-1}\right)$ \\ $\mathrm{x}, \mathrm{y}, \mathrm{z} \quad$ dimensionless Coordinates \\ x', y', z' Cartesian coordinates (m)
}

\section{Greek Symbols}

$\begin{array}{ll}\alpha & \text { magnetic field's angle with horizontal direction }\left(^{\circ}\right) \\ \alpha_{T} & \text { thermal diffusivity }\left(\mathrm{m}^{2} \cdot \mathrm{s}^{-1}\right) \\ \beta & \text { inclination angle of the cavity }\left(^{\circ}\right) \\ \beta_{\mathrm{T}} & \text { thermal expansion coefficient } \\ \beta_{\mathrm{C}} & \text { compositional expansion coefficient } \\ \lambda & \text { thermal conductivity of the fluid } \\ \lambda_{i} & \text { irreversibility distribution ratios, }(\mathrm{i}=1,2,3,4) \\ \mu & \text { dynamic viscosity of the fluid }\left(\mathrm{kg} \cdot \mathrm{m}^{-1} \cdot \mathrm{s}^{-1}\right) \\ \rho & \text { fluid density }\left(\mathrm{kg} \cdot \mathrm{m}^{-3}\right) \\ \sigma & \text { electrical conductivity }\left(\Omega^{-1} \cdot \mathrm{m}^{-1}\right) \\ v & \text { kinematics' viscosity }\left(\mathrm{m}^{2} \cdot \mathrm{s}^{-1}\right) \\ \Delta \mathrm{T}^{\prime} & \text { temperature difference }(\mathrm{K}) \\ \Delta \mathrm{C} & \text { concentration difference }\left(\mathrm{mol} \cdot \mathrm{m}^{-3}\right)\end{array}$

\section{Nomenclature}

B magnetic field (T)

C dimensionless concentration

C' concentration $\left(\mathrm{mol} \cdot \mathrm{m}^{-3}\right)$

$C_{h}^{\prime}$ hot side concentration $\left(\mathrm{mol} \cdot \mathrm{m}^{-3}\right)$

$C_{c}^{\prime}$ cold side concentration $\left(\mathrm{mol} \cdot \mathrm{m}^{-3}\right)$

$C_{0}^{\prime} \quad$ bulk concentration $\left(\mathrm{mol} \cdot \mathrm{m}^{-3}\right)$

$\mathrm{C}_{\mathrm{p}} \quad$ specific heat $\left(\mathrm{J} \cdot \mathrm{Kg} \cdot \mathrm{K}^{-1}\right)$

$\mathrm{D}$ mass diffusivity $\left(\mathrm{m}^{2} \cdot \mathrm{s}^{-1}\right)$

g gravitational acceleration $\left(\mathrm{m} \cdot \mathrm{s}^{-2}\right)$

$\mathrm{Gr}_{\mathrm{t}}$ thermal Grashof number

$\mathrm{Gr}_{\mathrm{c}}$ solutal Grashof number

$\mathrm{H}$ (L) height (length) of the cavity (m)

Ha Hartmann number

$\mathrm{Jk}$ diffusion Flux $(\mathrm{k}=\mathrm{u}, \mathrm{v}, \mathrm{T}, \mathrm{C})$

Le Lewis number

$\mathrm{N}$ Buoyancy ratio

\section{REFERENCES}

[1] J. A. Howard, P. A. Walsh and E. J. Walsh, Prandtl and capillary effects on heat transfer performance within laminar liquid-gas slug flows, Int. J. Heat and Mass Transfer 54, 21-22 (2011) 4752-4761

[2] S. Sarkar, A. Dalal and G. Biswas, Unsteady wake dynamics and heat transfer in forced and mixed convection past a circular cylinder in cross flow for high Prandtl numbers, Int. J. Heat and Mass Transfer 54, 15-16 (2011) 3536-3551

[3] C.D. Dritselis and N.S. Vlachos, Effect of magnetic field on near-wall coherent structures and heat transfer in magnetohydrodynamic turbulent channel flow of low Prandtl number fluids, Int. J. Heat and Mass Transfer 54, 15-16 (2011) 3594-3604

[4] Z. T. Yu, L.W. Fan, Y. C. Hu and K. F. Cen, Prandtl number dependence of laminar natural convection heat transfer in a horizontal cylindrical enclosure with an inner coaxial trian- 
gular cylinder, Int. J. Heat and Mass Transfer 53, 7-8 (2010) $1333-1340$

[5] Z. T. Yu, X. Xu, Y.C. Hu, L.W. Fan, K. F. Cen, Transient natural convective heat transfer of a low-Prandtl number fluid inside a horizontal circular cylinder with an inner coaxial triangular cylinder, Int. J. Heat and Mass Transfer 53, 23-24 (2010) 5102-5110

[6] M. O. Rouiss, L. R. Saad, G. Lauriat and A. Mazouz, Effect of Prandtl number on the turbulent thermal field in annular pipe flow, Int. C. Heat and Mass Transfer 37, 8 (2010) 958-963

[7] M. Bouabid, M. Magherbi, N. Hidouri and A.B. Brahim, Entropy Generation at Natural Convection in an Inclined Rectangular Cavity. Entropy 13 (2011) 1020-1033

[8] M. Bouabid, N. Hidouri, M. Magherbi and A.B. Brahim, Analysis of the Magnetic Field Effect on Entropy Generation at Thermosolutal Convection in a Square Cavity. Entropy 13 (2011) 1034-1054

[9] J.L. Lage and A. Bejan, The Ra-Pr domain of laminar convection in an enclosure heated from the side, Numer. Heat Transfer Part A Appl 19 (1991) 21-41

[10] J.M. Jalil and K.A. Al-Tae'y, MHD turbulent natural convection in a liquid metal filed square enclosure, Emirates Journal for Engineering Research 12 (2007) 31-40

[11] L. Davoust, R. Moreau and R. Bolcato, Control by a magnetic field of the instability of a Hadley circulation in a low-Prandtl-number fluid, Eur. J. Mech. B/Fluids 18 (1999) 621-634

[12] N.M. Al-Najem, K.M. Khanafer and M.M. .EL-Refaee, Numerical study of laminar natural convection in titled enclosure with transverse magnetic field, Int. J. Numer. Method.
H 8 (1998) 651-673

[13] M. Sankar, M. Venkatachalappa and I.S. Shivakumara, Effect of magnetic field on natural convection in a vertical cylindrical annulus $\|$, International Journal of Engineering Science 44 (2006) 1556-1570

[14] M. Sathiyamoorthy and Ali Chamkha, Effect of magnetic field on natural convection flow in a liquid gallium filled square cavity for linearly heated side wall(s), International Journal of Thermal Sciences 49 (2010) 1856-1865

[15] T. Pesso and S. Piva, Laminar natural convection in a square cavity: Low Prandtl numbers and large density differences, International Journal of Heat and Mass Transfer 52 (2009) $1036-1043$

[16] M. Magherbi, H. Abbassi, N. Hidouri and A.B. Ben Brahim, Second law analysis in convective heat and mass transfer. Entropy 8 (2006) 1-17

[17] H.J. Saabas and B.R. Baliga, Co-located equal-order control-volume finite-element method for multidimensional incompressible fluid flow, Part I: Formulation Numer. Heat Transfer Pt. B-Fund 26 (1994) 381-407

[18] S.V. Patankar, Numerical heat transfer and fluid flow, In Computational Methods in Mechanics and Thermal Sciences; Hemisphere/Mac Graw-Hill, New York, NY, USA (1980),

[19] H. Abbassi, S. Turki and S. Ben Nasrallah, Mixed convection in a plane channel with a built-in triangular prison, Heat Transfer Pt. A-Appl 39 (2001) 307-320

[20] H. Abbassi, S. Turki and S. Ben Nasrallah, Numerical investigation of forced convection in a plane channel with a built-in triangular prison, Int. J. Therm. Sci 40 (2001) 649-658

[21] L.C. Woods, The Thermodynamics of Fluid Systems, Oxford University Press: Oxford, UK (1975) 